\title{
NB-IoT Testbed for Industrial Internet of Things
}

\author{
Stephen Ugwuanyi \\ Electrical and Electronic Engineering \\ University of Strathclyde \\ Glasgow, United Kingdom \\ stephen.ugwuanyi@strath.ac.uk
}

\author{
Jidapa Hansawangkit \\ Electrical and Electronic Engineering \\ University of Strathclyde \\ Glasgow, United Kingdom \\ jidapa.hansawangkit@strath.ac.uk
}

\author{
James Irvine \\ Electrical and Electronic Engineering \\ University of Strathclyde \\ Glasgow, United Kingdom \\ j.m.irvine@strath.ac.uk
}

\begin{abstract}
The Internet of Things (IoT) is the general term for interconnecting objects interacting to achieve a common goal such as in the monitoring of physical phenomena and control of mission-critical infrastructures. The recent paradigm shifts in industrial communication technology necessitate that industrial parameters such as temperature, pressure, vibrations and leaks should be remotely monitored using reliable wireless technologies. They can provide a wide range of new service capabilities that would improve industrial operations and meet business needs. Examining the wireless technologies proposed to enable such connections, factors such as the infrastructural interoperability, cost of deployment, security and mobility need dictates the choice of wireless connections. Narrowband Internet of Things (NB-IoT) as an enabler of such services with massive connectivity options, good power utilization, longdistance transmission, higher data throughput in $4 \mathrm{G}$ and $5 \mathrm{G}$ applications has not been explored to a great degree. In this paper, we have shown an in-depth practical approach to setting up an NB-IoT test network and carried out different deployment testing using NB-IoT devices and an LTE/NB-IoT base station. The findings provide the necessary security requirements, the test network power and latency performance and the global spectrum deployment options for a potential private licensed NB-IoT network.
\end{abstract}

Keywords-Deployment, Industrial, Internet of Things, Narrowband, Security, Spectrum

\section{INTRODUCTION}

The Internet of Things (IoT) is a prime technology for connecting a huge number of electronic devices "things" remotely. Wi-Fi, Zig-bee, Bluetooth, Sigfox, LoRa, 4G/LTE and $5 \mathrm{G}$ are a few of the wireless technologies used for IoT [1]. Some of the reasons they are preferred over wired technology are as a result of the reduced installation/maintenance cost and mobility requirements but they are susceptible to vulnerabilities at the Radio Frequency (RF) interface. IoT technology came to light in the early-2000s and has significantly improved with the introduction of smart devices in mid-2007. It is projected to achieve a capacity of 50 billion nodes in 2020 [2] and over 100 billion devices in 2025 [3]. Furthermore, a simulation study by Ericsson claimed that NBIoT can provide support for 200,000 nodes per cell [4]. The realization of such a capacity is made possible following the global deployment of IPv6 communication protocol [5] and other innovations.

Industrial automation, remote processing and monitoring control, telemetry, data management and protection are few areas where these innovations are already taking place in different magnitudes. "Industrial machinery, transportation monitoring, logistics/asset tracking, healthcare, intelligent buildings, smart agriculture and smart metering" are specific sectors where IoT is the catalyst for the development [6]. IoT has generally improved beyond the level where only mobile devices and computers were linked to the internet [7]. Internet connectivity now includes 'things' such as industrial sensors

This research is funded by the Nigerian Petroleum Technology Development Fund (PTDF) award number PTDF/ED/PHD/USO/1092/17 and actuators, home equipment and other personal handheld devices.

\section{NB-IOT TECHNOLOGY}

Narrowband Internet of Things (NB-IoT) is a Long-Term Evolution (LTE) based Radio Access (RA) technology for Low Power Wide Area Network (LPWAN) IoT connectivity and services. It is part of the new RA cellular technology in the Third Generation Partnership Project (3GPP) Release 13, $14,15,16$ offerings and in 17 Release due in 2021 [8]. NBIoT is increasingly used to support machine-type legacy Global System for Mobile Communication (GSM), Long Term Evolution (LTE) and $5^{\text {th }}$ Generation (5G) communication systems. The choice of NB-IoT for creating a network of sensors and other ultra-low IoT devices is based on the enhanced coverage, deployment flexibility options, deep penetration and reduced energy consumption (Power Save Mode (PSM) and Extended Discontinuous Reception (eDRx)) and the cellular IoT Evolved Packet System (EPS) optimization. The flexibility in the deployment options come from the wide range of frequency bands LTE networks can be deployed in. For instance, the minimum frequency of $180 \mathrm{kHz}$ requirements for both uplink and downlink makes it possible to use one GSM carrier $(200 \mathrm{kHz})$ or one Physical Resource Blocks (PRBs) existing within an LTE network for NB-IoT deployment [9].

NB-IoT is a class of licensed LPWAN, a general term for licensed or unlicensed small data transfer protocols. LoRaWAN is an open and LoRa Alliance standardized LPWAN. LoRaWAN features in remote monitoring applications have been investigated within the same research group; Centre for Dynamic Intelligent Communication (CIDCOM), University of Strathclyde. The study, which used a prototype fault passage indicator to monitor voltage faults along an $11 \mathrm{kv}$ power line shows that LoRaWAN was suitable for remote applications of low data rate. NB-IoT was identified as a step further from LoRaWAN and Sigfox [10]. The 2019 Berlin LoRA Alliance event further reveals how LPWAN is enabling IoT across all sectors [11].

Comparing the coverage and other capabilities of licensed and unlicensed LPWAN technologies, [12] and [13] demonstrated that NB-IoT has good penetrating power in all deployment modes with maximum coupling loss performance of $164 \mathrm{~dB}$. It also has better coverage, including indoor applications. These in addition to other advantages have increased NB-IoT applications and services among Mobile Service Providers (MSP) and Network Professionals (NP) as a suitable protocol for the emerging IoT market. It is a clear indication that NB-IoT which continues to evolve from GSM/LTE/5G networks, enabling good connectivity and services for a wide range of devices is a technology of future IoT network.

NB-IoT can be deployed by a software upgrade to an existing GSM and LTE network infrastructure, reallocating 


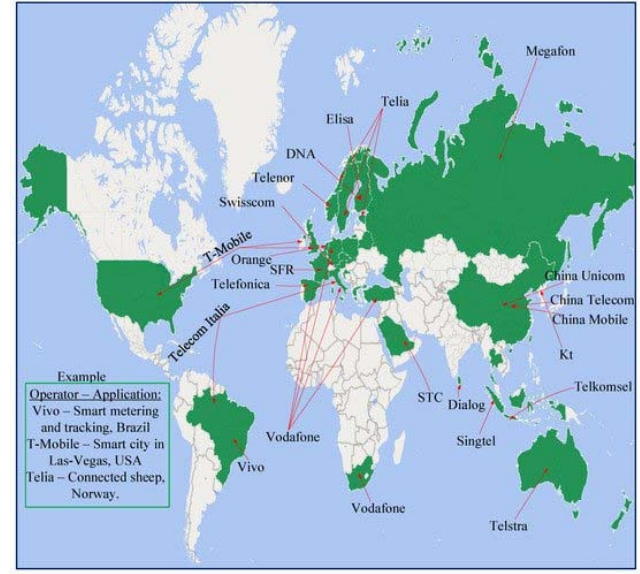

Fig. 1. Global NB-IoT Deployment in 2019 [14]

available frequency band possibly from GSM, and the use of features in the new $5 \mathrm{G}$ design. The global deployment of NBIoT, as shown in Fig. 1 is facilitated by the mobile network provider within the regions around the world. A standalone NB-IoT network has been launched in Florida by Puloli using the $700 \mathrm{MHz}$ A-Block spectrum to provide services to utilities and other parallel industries. Other identifiable use cases include the sea, agriculture, smart city, smart metering and tracking with major equipment manufacturers to play a major role in improving the network security [14]. NB-IoT application in the streams of oil and gas sector has the potential to track and reduce the number of wastages, but its global visibility is comparatively very small with other technologies.

\section{A. NB-IoT Deployment Modes}

Narrowband IoT has three modes of deployment options as presented in Fig. 2. The standalone deployment, which is considered the most expensive, requires the installation of new network resources like in the case of GSM and skills to develop and deploy. On the other hand, NB-IoT deployment within an LTE network can be in either the in-band or guardband mode, hence, having the same LTE design features such as security. The guard-band and in-band deployment options uses the $180 \mathrm{kHz}$ single PRB of LTE core network and enables the support for Narrowband Uplink Shared Channel (NPUSCH) allocation. Narrowband Physical Downlink Shared Channel (NPDSC) and the NPUSCH carries data packets for downlink (DL) and uplink (UL) respectively.

Standalone: Independently deployed as a dedicated carrier using spectrum greater than the required $180 \mathrm{kHz}$ frequency bandwidth. GSM wireless access networks and satellite communication systems may be used to deploy NBIoT in the standalone mode. This is achieved by refarming certain aspect of the frequency spectrum to include $200 \mathrm{kHz}$ and $100 \mathrm{kHz}$ guard-band for different operators and the same operator respectively deploying both GSM and LTE [15].

Guard-band: It is also possible to deploy NB-IoT within the existing LTE networks using the LTE guard-band. The

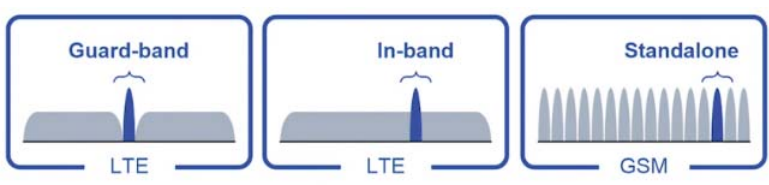

Fig. 2. NB-IoT Deployment Modes
LTE channel bandwidth is not fully occupied. The 5\% unused resource blocks guard bands are used to deploy NB-IoT between LTE carriers.

In-band: Deployed in the PRB of an LTE carrier wave. Free LTE PRB is reused, making the deployment more advantageous for maintenance [16]. This is the operational mode on which this study was carried out. However, similar testing was also performed in standalone and guard-band modes.

\section{B. Benefits of NB-IoT}

The wider benefits of using NB-IoT technology in monitoring and control applications as observed in this study are not limited to those shown in Fig. 3.

High data rate: Unlike in LoRa and Sigfox technologies where the maximum data rate is $84 \mathrm{msg} /$ day and $140 \mathrm{msg} /$ day respectively, NB-IoT maximum packet size and data rate depends on the network provider offerings and capability of the NB-IoT devices. The maximum LoRa uplink and downlink packet size of the FPI project were 62 bytes [10]. Sigfox has a data rate between 0.1 to $0.6 \mathrm{kbps}$ and a maximum packet size of 12 bytes uplink and 8 bytes downlink. The data rate for LTE-M and NB-IoT are usually up to $1 \mathrm{Mbps}$ and 375 kbps respectively, and subject to improvement with the emergence of new NB-IoT Releases.

Improved coverage: For applications in the hard-to-reach locations such as in the sea and land oil rigs and platforms located miles away with a localized gateway, coverage enhancement is achieved by using repetition transmission of up to 128 and 2048 for UL and DL respectively but at a reduced data rate [14]. The indoor extended coverage of NBIoT is within the range of $20 \mathrm{~dB}$ and maximum coupling loss of $20 \mathrm{~dB}$ better than the LTE network. NB-IoT relies on $4 \mathrm{G}$ infrastructures to deliver wide coverage to indoor and dense locations with good response rate surpassing that of LoRa to reach $20 \mathrm{~km}$ at low frequencies below $1 \mathrm{GHz}$. A software upgrade is one of the coverage enhancement options possible of achieving $100 \mathrm{~km}$ in special use cases.

Latency insensitivity: Latency is not a priority in the NBIoT network, but the technology can accommodate a maximum delay of $10 \mathrm{~ms}$ for error delivery in applications such as the alarm systems [17], [9]. The delay in packet delivery is generally due to the mode of transmission, the frequency of transmission, the timing, a process of cell acquisition, etc. Early Data Transmission (EDT) and Hybrid Automatic-Repeat-Request (HARQ) are new schemes developed to reduce the repetition rate and guarantee successful transmissions.

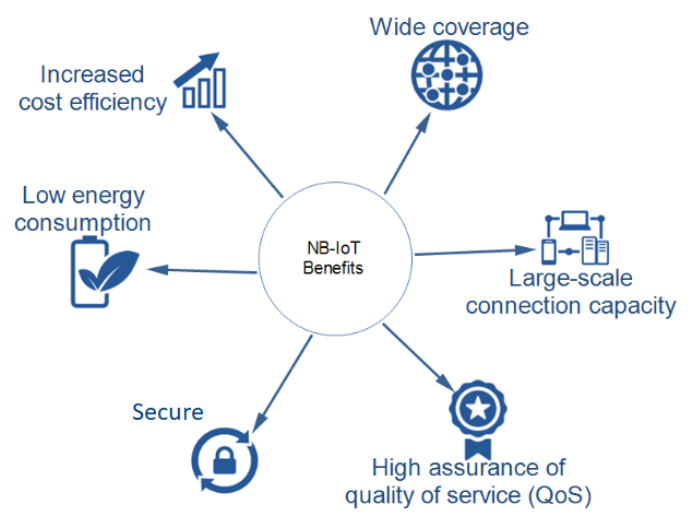

Fig. 3. Benefits of NB-IoT Technology 
Long battery life: NB-IoT targets applications with a long battery life of up to 10 years at a maximum coupling loss (MCL) of $164 \mathrm{~dB}$. The reduced power backoff also helps to improve battery efficiency. The User Equipment (UE) goes into PSM in the inactivity period while attached to the base station and cannot be pinged by the eNodeB. The inactivity period of 413 days in Release 13 extends the battery life. In eDRx mode, the UE is in an inactive mode for a small period and synchronized to wake up by the sensor push data, an aspect controllable by the software implementation.

Possible firmware update: Firmware Over-The-Air (FOTA) is still a challenging task to realize in the NB-IoT network. The downlink transmission rate is very low in recent designs and cannot support software updates. This goes further to affect the mobility of the devices as roaming will also be difficult to achieve.

Massive connectivity: NB-IoT supports a very high number of UEs in one single carrier. With the help of the Resource Unit (RU) allocation, UEs are allocated to an RU where one UE occupies $3.75 \mathrm{kHz}$ or $15 \mathrm{kHz}$ UL bandwidth and $15 \mathrm{kHz}$ for DL bandwidth.

Less complex and reduced cost: The low-cost attribute of NB-IoT is attached to the UEs low storage capacity, the reduced frequency of operation, single antenna design and the modulation scheme limitations. The less complex UEs are the more packet delay, more channel interference and less estimation introduced in the transmission link.

\section{RELATED WORK}

Narrowband IoT technology is new and the aspects regarding functional testing and deployment are few and not readily available. Unlicensed LPWAN technologies, such as the Sigfox and LoRa, were the preferred choices for communication infrastructure requiring good coverage, smaller data rate transmission and has been available longer than NB-IoT. Sigfox and LoRa are exempt from licensing and operate in the Industrial, Scientific and Medical (ISM) radio band of $868 \mathrm{MHz}$ in Europe. However, studies exist with frameworks of enabling unlicensed NB-IoT (NB-IoT-U) in the Sub-1GHz [18], [19].

Security was their greatest challenge until the emergence of NB-IoT in the 3GPP Release 13, initiated by Huawei and Vodafone in 2014 as a cellular Narrowband Machine to Machine (NB-M2M) technology. The 3GPP specifications provides important security and other attributes in the implementation of the NB-IoT network. The list is not limited to key type, key management, data freshness, authorization, availability, data integrity, authentication and confidentiality.
Literature focusing on NB-IoT deployment options are few and most of the studies are currently pilot testing. Vodafone, Huawei and Deutsche Telekom have test beds in various locations [4] globally. Three UK is also running pilot LPWA network tests to help utilities improve their service performance, detect faults and determine their carbon footprint [20]. These studies have raised research questions in the aspects of power utilization, battery life, price of the modules, the data-carrying capacity, coverage issues, radio frequency management, mobility management, scheduling, backward compatibility and a host of other variables [14].

However, work is in the area of extending NB-IoT coverage [21] and [12]; co-existence of NB-IoT in LTE environment [22] and [6]; NB-IoT design and specifications [23] and [9]; and applications [24], [8] and [9]. The effect of LTE and NB-IoT to exist in one cellular network resource as Junghoon and Hoyoung investigated [22], identified a significant interference and a consequence of a reduction in the network performance. The study found that the use of 15 $\mathrm{kHz}$ and $3.75 \mathrm{kHz}$ subcarrier frequencies do not eliminate interference completely but seem to be more pronounced in the $3.75 \mathrm{kHz}$ as against the LTE $15 \mathrm{kHz}$ standard.

\section{METHODOLOGY}

Our NB-IoT test network setup, see Fig. 4, employed a functional Amarisoft wireless protocol stack based LimeSDR (LMS7002M) hardware as the radio front-end to generate the standard core LTE and NB-IoT base station physical signals on the same processor. One of the University of Strathclyde's 5G RuralFirst project devices used in addressing the UK's digital divide. It is a Multiple-Input Multiple-Output (MIMO) transceiver based on Field-Programmable Radio Frequency (FPRF) for hardcore Digital Signal Processing (DSP), low cost and wide frequency $(100 \mathrm{kHz}$ to $3.8 \mathrm{GHz})$ module that runs on Snappy Ubuntu Core, see Table 1 for test parameters. The Pycom module (Fipy) attached to a Pycom Pysense board and Expansion Board 3.0 is the IoT Node. The Fipy is a new independent node scale IoT project-based board programmable in the Visual Studio Code and Atom. It has Cellular, WiFi, Sigfox, Lora, Bluetooth and LTE CAT M1/NB1 wireless technology options.

To obtain the parameters needed to configure the Random Access Channel (RACH) procedure, the UE scans and detects synchronization signals and reads System Information Block (SIB). The Narrowband Primary Synchronization Signal
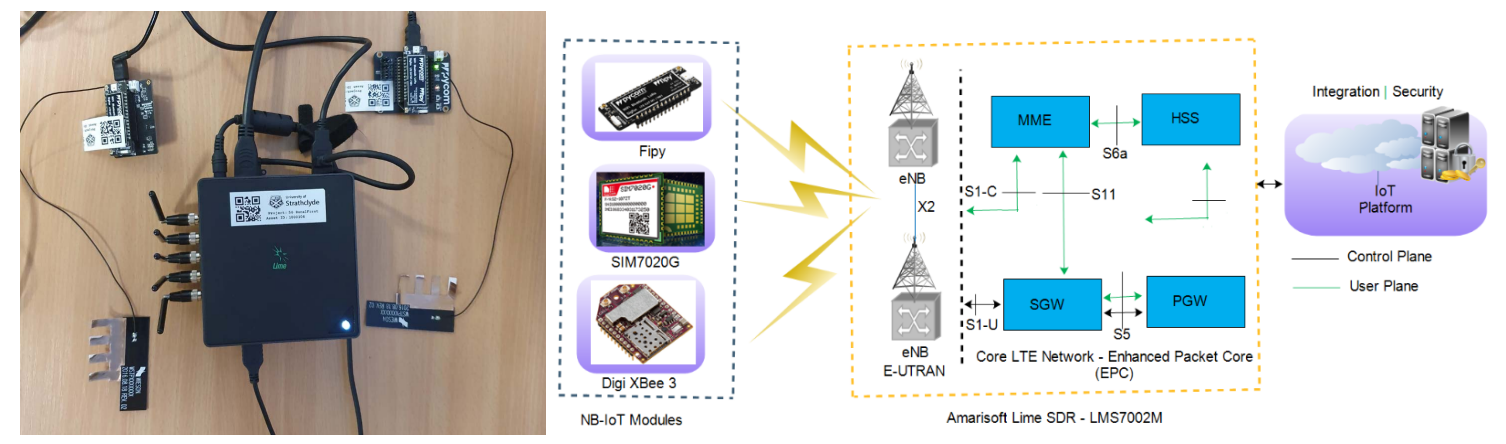

Fig. 4. NB-IoT Experimental Setup 


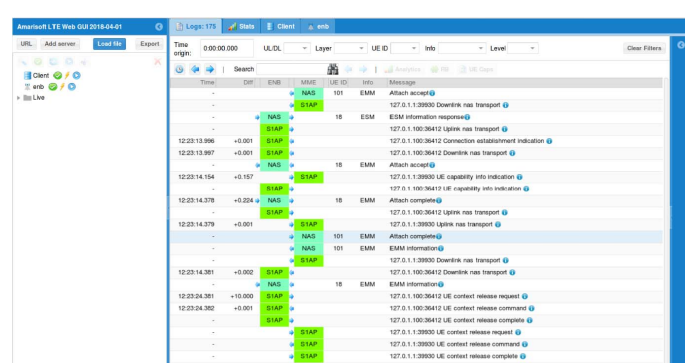

Fig. 5. NB-IoT Network Attachment Procedure

(NPSS) for timing and modulation information and Narrowband Secondary Synchronization Signal (NSSS) for cell information of the base station. The decoding of Memory Information Block (MIB) enable the UE to identify the System Frame Number (SFN), Hyper Frame Number (HFN), UL and DL information. With this information, the frequency hopping pattern is generated at the UE which is then used to identify the frequency carrier that Narrowband Physical Random Access Channel (NPRACH) lies and which is used to perform initial access to the NB-IoT network and request transmission resources. For the UE to know the selected carrier and the transmission time allocated to Narrowband Physical Random Access Control Channel (NPRACH), UE is configured for DL transmission, the Narrowband Physical Downlink Shared Channel (NPDSCH) and Narrowband Physical Uplink Shared Channel (NPUSCH) for UL transmission. The UE decodes the Memory Information Block

Algorithm 1. UE Connection Establishment Procedure

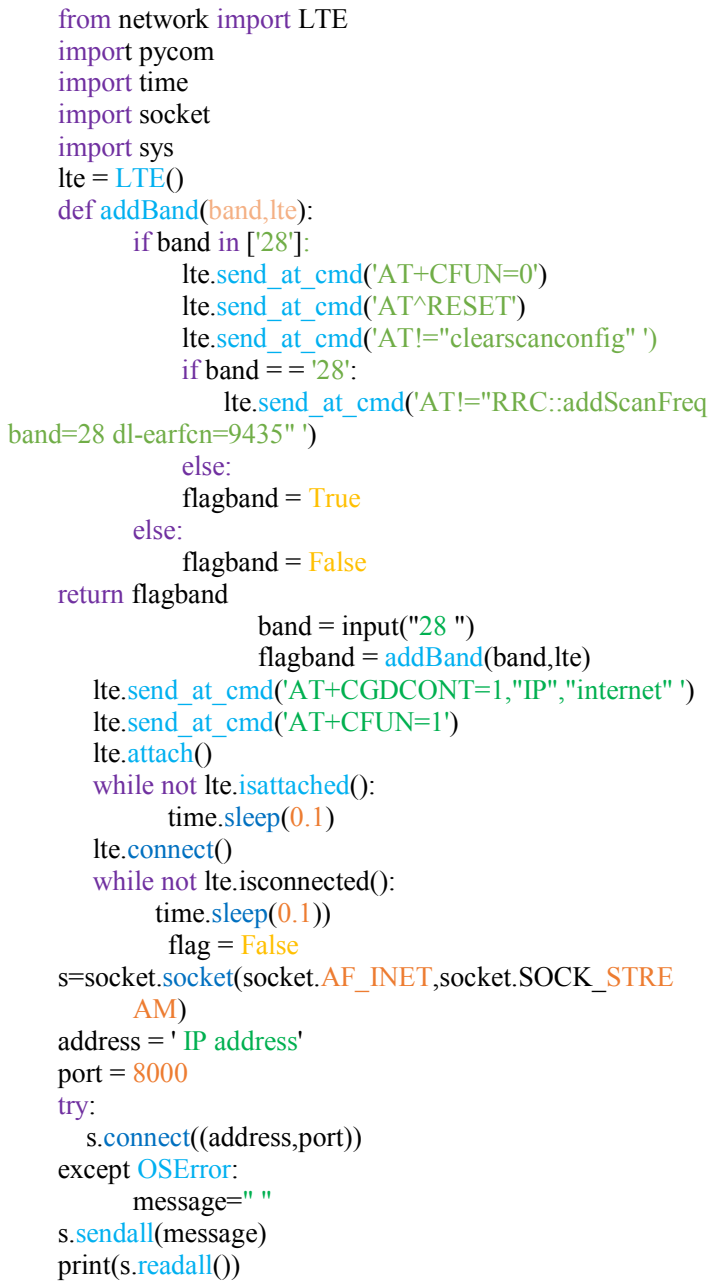

Table 1. Test Parameters

\begin{tabular}{|l|c|}
\hline \multicolumn{1}{|c|}{ Test Parameters } & Values \\
\hline Sample rate & 30 \\
\hline Dec_Inter & 2 \\
\hline Rx-power & $-15 \mathrm{dBm}$ \\
\hline Tx-power & $-3 \mathrm{dBm}$ \\
\hline Bandwidth & $180 \mathrm{kHz}$ \\
\hline Frequency carrier & Band $28(\mathrm{dl}$-earfcn $=9435)$ \\
\hline
\end{tabular}

(MIB) for scheduling information and System Information Block 1 (SIB1) for system information types (mapping, periodicity, window length, etc.) respectively and broadcasted at intervals.

While the NB-IoT EPC exchange control messages with the NAS of UEs and forward IoT data to the platform for processing, the eNB communicates the network access messages to the higher layer and handles cell management via the S1 interface. The IoT platform shown in Fig. 5 is the core network data visualization interface for varied UEs, the eNB and core network (MME) data analytics and communication procedure.

Implementing 3GPP NB-IoT Release 13, the network performance can be measured in terms of the extended indoor coverage, increasing the number of IoT devices supported in a cell-site, reducing the complexity of adding more devices to the network, improving the power utilization and overall latency [17]. Cost, power utilization and latency is the focus of this paper. The 3GPP Release 14 introduced enhanced location tracking, support for multicast downlink transmission, new power class(es) for UEs, multi-carrier operations and non-anchor carrier support [25]. The 3GPP release 15 optimizes the support for EDT during the RA connection procedure. Release 16 covers the enhancement in the 3GPP standardization Release 15 5G New Radio (5GNR) to include the self-organizing ability, the ability to coexist with NR, mobility, scheduling, transmission and equipment enhancement.

\section{A. NB-IoT Devices}

The SIMCom, Fibocom and Digi NB-IoT modules are other flexible cellular hardware IoT devices with good NBIoT performance metrics such as data throughput and battery life. The bands, security supported, data rate and price of each device compared with Fipy is shown in Table 2 outweighing others in price, security capabilities and data rate. The bands in green are the current global deployable bands supported by the devices. Vodafone has already commercialized NB-IoT network using the $800 \mathrm{MHz}$ band in the west part of the UK [20]. 
Table 2. Table of NB-IoT Devices

\begin{tabular}{|c|c|c|c|}
\hline Devices & Bands & $\begin{array}{l}\text { Support } \\
\text { Features }\end{array}$ & $\begin{array}{c}\text { Price } \\
\text { Jan.2020 }\end{array}$ \\
\hline $\begin{array}{l}\text { Pycom } \\
\text { (Fipy) }\end{array}$ & $\begin{array}{l}1,2,3,4,5,8, \\
12,13,18,19, \\
20 \text { and } 28\end{array}$ & $\begin{array}{l}300 \mathrm{kbps} \mathrm{DL} \text { and } \\
375 \mathrm{kbps} \mathrm{UL} \text { in } \\
1.4 \mathrm{MHz}, 40 \mathrm{kbps} \\
\mathrm{DL} \text { and } 55 \mathrm{kbps} \\
\mathrm{UL} \text { in } 200 \mathrm{kHz} \text {, } \\
\text { SSL/TLS } 1.2 \text {, } \\
\text { WPA, AES. }\end{array}$ & $\$ 46.89$ \\
\hline $\begin{array}{c}\text { Digi } \\
\text { (Digi XBee 3) }\end{array}$ & $\begin{array}{l}1,2,3,4,5,8 \\
12,13,18,19 \\
20,25,26,28 \\
\text { and } 30\end{array}$ & $\begin{array}{l}\text { Multiple band, up } \\
\text { to } 27.2 \mathrm{kbps} \mathrm{DL} \\
\text { and } 62.5 \mathrm{kbps} \mathrm{UL}\end{array}$ & $\$ 69.00$ \\
\hline $\begin{array}{c}\text { SIMCom } \\
\text { (SIM7020G) }\end{array}$ & $\begin{array}{l}1,2,3,4,5,8, \\
12,13,17,18, \\
19,20,25,26, \\
28,66,70 \text { and } \\
71\end{array}$ & $\begin{array}{l}\text { Global bands, } \\
\text { 3GPP Release } 13, \\
14,150 \mathrm{kbps} \text { UL, } \\
100 \mathrm{kbps} \text { DL, TLS }\end{array}$ & $\$ 69.00$ \\
\hline $\begin{array}{c}\text { Fibocom } \\
\text { (M910-GL) }\end{array}$ & $\begin{array}{l}1,2,3,4,5,8 \\
12,13,18,19 \\
20,26,28 \text { and } \\
39 \text { on TDD }\end{array}$ & $\begin{array}{l}32 \text { kbps } \text { DL/70 } \\
\text { kbps UL, eSIM, } \\
\text { IPv6, TCP, UDP }\end{array}$ & $\$ 109.00$ \\
\hline
\end{tabular}

\section{RESULTS A ND DISCUSSIONS}

\section{A. Mobility Management Entity (MME)}

The NB-IoT MME test network has the Serving Gateway (SGW), Packet Data Network Gateway (PGW) and Home Subscriber Server (HSS) in-built. Given a theoretical data rate of $151 \mathrm{Mbps}$ for $20 \mathrm{MHz}$ cell in LTE, eNodeB resources of $200 \mathrm{kHz}$ is shared among the IoT modules supporting all operation modes. Line of Sight (LoS) and LTE band interference affected the overall throughput of the transmission channel. To provide a software-defined configurable NB-IoT test network, the LTE core network interface (LTEMME) is attached to LTEENB on the same processing machine using the $\mathrm{S} 1$ interface. The list of registered UEs registered and attached to the eNodeB is shown in Fig. 6 . The pycom UE tolerated integrity checks and Advanced Encryption Standard (AES).

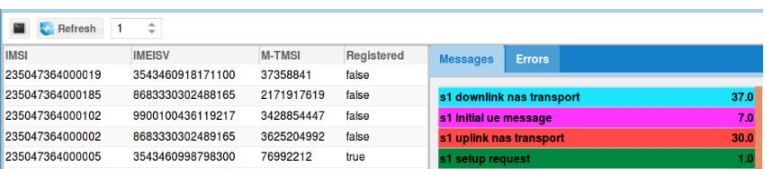

Fig. 6. MME Interface showing UEs attached to the Network

\section{B. Long Term Evolution Evolved Node B (LTEENB)}

LTEENB is a software-based base station running Stream Control and Transmission Protocol (STCP) on 5th generation or higher core processors configurable to support NB-IoT cells. The radio front end performs baseband signal conversion generated by the processor before being interfaced with the LTE standard core network (MME) interface at S1 plane. The $15 \mathrm{kHz}$ and $3.75 \mathrm{kHz}$ subcarrier spacing, single and multi-tone category NB1 and NB2 UEs were supported. The eNodeB is responsible for optimizing the radio interface by assigning the radio network temporary identifier to the UE to distinguish radio UEs and radio channels. The eNodeB also helped to reduce the collision in a multi-user transmission environment. UEs antenna was kept a few meters away from the LMS7002M antenna and bands greater than band 7 is recommended if interference is to be kept very low.

\section{Latency}

Finding the transmission latency of NB-IoT UEs is an important way of ascertaining the network performance. Latency exist in IoT network as a result of large number of connected devices and channel conditions. More specifically, they include mode of transmission, modulation scheme, error recovery pattern, propagation delay, low device complexity, queuing management, timing and frequency requirements. NB-IoT network can tolerate a latency value of $10 \mathrm{~ms}$ [14]. With few UE being attached to the test network, latency here was based on carrying out a faster RRC release and the UE transmitting data during the RACH procedure which reduced latency and power consumption.

\section{Power Consumption}

The functionality of UEs on the NB-IoT test network comprised of the Lime microsystems SDR front-end and the performance of the entire system. The energy efficiency of NB-IoT technology is aimed at providing long battery life of up to 10 years for a coupling loss of $164 \mathrm{~dB}$. NB-IoT modules provides machine-to-machine communication either through direct power source or battery. However, the 3GPP higher Releases incorporate Power Saving Mode (PSM) and Extended Discontinuous Reception (eDRx) options to improve IoT device battery life. The improvement in the new releases allows the UEs to transmit data even during the Radio Resource Control (RRC-idle) state as a way of reducing power consumed during transmission and reception. The method of waking the UEs up from deep sleep mode also improves the power consumption.

The average current measurements obtained when the IoT module scans the band and registered on the network in band 28 and sends a GET request message is $151 \mathrm{~mA}$ and $126 \mathrm{~mA}$ respectively. An average of $3 \mathrm{mAh}$ is an extra energy needed to reconnect the module to the network while $1.8 \mathrm{mAh}$ was needed for an uplink message. This, however, is observed to deviate largely from $100 \mu \mathrm{Ah}$ average power consumed by the same module when joining a LoRa network and sending a few approximately 168 bytes of uplink data for spread factors of 7 and 12 . The battery life reduces significantly as a result of the constant retransmission especially when the UE was a few meters away from the eNB. When the application requires data update more frequently, the energy consumed became greater. To improve the power consumption of the UEs, other efficient options of energizing the modules independently should be explored.

\section{E. NB-IoT Security}

NB-IoT is characterized by low power consumption devices with low processing power and lighter UE security complexity. Security in NB-IoT then relates to hardening of IoT products, securing communication links, complying with international security standards (3GPP), enforcing management policy, trusting of third-party services and software, key management issues and embedded security techniques. The perception layer is critical to the security of NB-IoT network. The inefficiency of the devices means that attacks are imminent in the event of a network cyber incident. Following security classifications in [26], NB-IoT security is classified into perception, transmission, and application layers.

The 3GPP documentation on which this study is based specifies the security requirements between UEs and the NBIoT network. In [27], NB-IoT security relied on SNOW 3G 
encryption to provide data confidentiality and ATR-128 provides secure inter-mobile network data transfer. In this study, AES-128,192,256 is the Fipy supported security for encrypting the sensor payload before transmission, guaranteeing NB-IoT security attributes such as privacy, confidentiality, data freshness, authorization and integrity as they are crucial in all sensitive IoT use cases. Hence, the need for further study on security especially in the NB-IoT-U.

\section{FUTURE DIRECTION}

The use of certain wireless technology for IoT connectivity is pervasive. In early 2020, the number of commercial NB-IoT networks globally have increased from 45 in 2018 [27] to 92 [28] with no visibility in many countries. LTE and $5 \mathrm{G}$ networks provide suitable platforms for mobile NB-IoT development and require minimal technical changes such as a software upgrade by the Mobile Network Operators (MNOs). Inheriting the design features of $4 \mathrm{G}$ and $5 \mathrm{G}$, it is considered more secure than other LPWAN technologies and suitable for pervasive applications.

As the demand for connectivity continues to increase in NB-IoT ecosystem, we will expand this platform to include other IoT technologies such as LTE-M in order to support all kinds of IoT devices. Other research needs include delivering backward compatibility and interoperability issues while ensuring adequate security. Also, finding robust algorithm to manage mobility and software update in the network while maintaining UE complexity very low is another future research direction.

\section{CONCLUSION}

This work presented a practical deployment testing of a typical industry NB-IoT network, outlining the current trends in NB-IoT development. We demonstrated that NB-IoT is a more appropriate technology for massive industrial remote applications. The study also provides early testing insight on IoT UE power utilization, cost, latency and what equipment standard security requirements are for NB-IoT deployment in the face of a cyber-connected world. However, the frequency of operation and license is a critical parameter as one must have the legal right to transmit on any LTE frequency in a country and be sure to ensure reduced interference through the deployment of evaluated NB-IoT devices.

\section{REFERENCES}

[1] D. C. Sicker, "Policy and Regulatory Issues," IEEE Internet Things Mag., vol. 2, no. 3, pp. 2-3, Sep. 2019.

[2] M. Martonosi, "Keynotes: Internet of Things: History and hype, technology and policy," 2016 49th Annu. IEEE/ACM Int. Symp. Microarchitecture, pp. 1-2, 2016.

[3] S. Sibiya and O. O. Olugbara, "Reliable internet of things network architecture based on high altitude platforms," 2019 Conf. Inf. Commun. Technol. Soc. ICTAS 2019, pp. 1-4, 2019.

[4] T. Winchcomb, S. Massey, and P. Beastall, "Review of latest developments in the Internet of Things Client: Ofcom Riverside House 2a Southwark Bridge Road London SE1 9HA," 2017. G. Mulligan, "IPv6 FOR IoT AND GATEWAY," in Internet of Things and Data Analytics Handbook, Hoboken, NJ, USA: John Wiley \& Sons, Inc., 2016, pp. 187-196.

[6] J. Navarro-Ortiz, S. Sendra, P. Ameigeiras, and J. M. Lopez-Soler, "Integration of LoRaWAN and $4 \mathrm{G} / 5 \mathrm{G}$ for the Industrial Internet of Things," IEEE Commun. Mag., vol. 56, no. 2, pp. 60-67, 2018. U. S. Clusters, "Cyber security," p. 60, 2010.

[8] 3GPP, "Release 16," 2019. [Online]. Available: https://www.3gpp.org/release-16. [Accessed: 26-Sep-2019].
[9] Y. P. E. Wang et al., "A Primer on 3GPP Narrowband Internet of Things," IEEE Commun. Mag., vol. 55, no. 3, pp. 117-123, 2017.

[10] R. Mcpherson, C. Hay, J. Irvine, and S. Member, "Using LoRaWAN Technology To Enhance Remote Power Network Monitoring," 2019 IEEE 89th Veh. Technol. Conf., pp. 1-5, 2019.

[11] L. Alliance, "LoRa Alliance ${ }^{\mathrm{TM}}$ Bringing LoRaWAN® Live! to Berlin; Biggest LoRaWAN Event of the Year will Showcase Value of LoRaWAN Deployments and Certification." [Online]. Available:https://www.globenewswire.com/newsrelease/2019/05/02/1815427/0/en/LoRa-Alliance-BringingLoRaWAN-Live-to-Berlin-Biggest-LoRaWAN-Event-of-theYear-will-Showcase-Value-of-LoRaWAN-Deployments-andCertification.html. [Accessed: 02-Feb-2020].

[12] B. Vejlgaard, M. Lauridsen, H. Nguyen, I. Z. Kovacs, P. Mogensen, and M. Sorensen, "Coverage and Capacity Analysis of Sigfox, LoRa, GPRS, and NB-IoT," IEEE Veh. Technol. Conf., vol. 2017-June, 2017.

[13] M. Lauridsen, H. Nguyen, B. Vejlgaard, I. Z. Kovacs, P. Mogensen, and M. Sorensen, "Coverage Comparison of GPRS, NB-IoT, LoRa, and SigFox in a $7800 \mathrm{~km}$ Area," IEEE Veh. Technol. Conf., vol. 2017-June, pp. 2-6, 2017.

[14] C. B. Mwakwata, H. Malik, M. Mahtab Alam, Y. Le Moullec, S. Parand, and S. Mumtaz, "Narrowband Internet of Things (NBIoT): From Physical (PHY) and Media Access Control (MAC) Layers Perspectives," Sensors, vol. 19, no. 11, p. 2613, Jun. 2019.

[15] J. L. R. Sarmiento, "Interfeence Management in NB-IoT for Heterogeneous Wireless Network". PhD Thesis, Tallinn University of Technology, 2019.

[16] E. Shin and G. Jo, "Structure of NB-IoT NodeB system," in 2017 International Conference on Information and Communication Technology Convergence (ICTC), 2017, pp. 1269-1271.

[17] N. Mangalvedhe, R. Ratasuk, and A. Ghosh, "NB-IoT deployment study for low power wide area cellular IoT," IEEE Int. Symp. Pers. Indoor Mob. Radio Commun. PIMRC, no. 1, 2016.

[18] R. Sun, S. Talarico, W. Chang, H. Niu, and H. Yang, "Enabling NB-IoT on Unlicensed Spectrum," in IEEE International Symposium on Personal, Indoor and Mobile Radio Communications, PIMRC, 2019, vol. 2019-September.

[19] R. Sun, W. Chang, S. Talarico, H. Niu, and H. Yang, "Design and performance of unlicensed NB-IoT," in Proceedings of the International Symposium on Wireless Communication Systems, 2019, vol. 2019-August, pp. 469-473.

[20] Ofcom, "Connected nations 2019 - uk report," https://www.ofcom.org.uk/ data/assets/pdf file/0023/186413/C onnected-Nations-2019-UK-final.pdf, 2020. Last accessed 02 April 2020.

[21] S. Ha, H. Seo, Y. Moon, D. Lee, and J. Jeong, "A novel solution for NB-IoT cell coverage expansion," 2018 Glob. Internet Things Summit, GIoTS 2018, pp. 1-5, 2018.

[22] J. Oh and H. Song, "Study on the Effect of LTE on the Coexistence of NB-IoT," Int. Conf. Ubiquitous Futur. Networks, ICUFN, vol. 2018-July, pp. 610-612, 2018.

[23] Y. Miao et al., "Narrowband Internet of Things: Simulation and Modeling," IEEE Internet Things J., vol. 5, no. 4, pp. 2304-2314, 2018.

[24] V. Nair, R. Litjens, and H. Zhang, "Assessment of the Suitability of NB-IoT Technology for ORM in Smart Grids," 2018 Eur. Conf. Networks Commun. EuCNC 2018, pp. 418-423, 2018.

[25] R. Ratasuk, N. Mangalvedhe, Y. Zhang, M. Robert, and J.-P. Koskinen, "Overview of narrowband IoT in LTE Rel-13," in 2016 IEEE Conference on Standards for Communications and Networking (CSCN), 2016, pp. 1-7.

[26] M. Chen et al., "Narrow Band Internet of Things," vol. 3536, no. c, pp. 1-19, 2017.

[27] K. K. Nair, A. M. Abu-Mahfouz, and S. Lefophane, "Analysis of the narrow band internet of things (NB-IoT) technology," 2019 Conf. Inf. Commun. Technol. Soc. ICTAS 2019, pp. 1-6, 2019.

[28] GSMA, "Mobile iot network launches," https://www.gsma.com/iot/mobile-iot-commercial-launches/, 2020. Last accessed 31 January 2020. 